\title{
Original
}

\section{Polimorfimos en genes de respuesta inflamatoria en cáncer renal metastásico}

\author{
Pablo Sáenz López*, Fernando Vázquez Alonso**, José M. Romero*, Rafael Carretero*, \\ Miguel Tallada Buñuel**, Francisco Ruiz Cabello*, José Manuel Cózar Olmo** \\ *Servicio de Análisis Clínicos e Inmunología. **Servicio de Urología. Hospital Universitario Virgen de las \\ Nieves, Granada, España
}

\section{Resumen}

La inflamación se ha implicado como un factor etiológico en varios tipos de cánceres humanos. Las variaciones alélicas de los genes implicados en la inflamación son candidatos como determinantes genéticos del riesgo de carcinoma renal. El propósito de los estudios realizados fué investigar si los polimorfismos de genes que llevan a un incremento de los niveles de citoquinas proinflamatorias y quimioquinas están asociados con un aumento en el riesgo de carcinoma renal. Para ello diseñamos varios estudios caso-control para probar la asociación entre el carcinoma renal y los polimorfismos IL10-1082 A/G (rs 1800896), IL10-592 A/C (rs 1800872), IL10-819 C/T (rs 1800871), IL10-1082 A/G, IL4-590 C/T (rs 2243250), TNF-A -308 A/G (rs 1800629), RANTES -403 G/A (rs 2107538), IL1-A -889 C/T (rs 1800587), MCP-1 2518 G/A (rs 1024611) y CTLA-4/ +49 A/G (rs 231775) y CTLA-4 CT60 A/G (rs 3087243) en 127 pacientes de carcinoma renal, y como control 176 personas sanas.

Los resultados obtenidos de los polimorfismos de la citoquina IL-10-1082 A/G fueron que el estado de heterocigosidad AG presenta el factor de riesgo principal en relación con estadio tumoral localmente avanzado o metastasico en el carcinoma renal. En el caso de la molécula de CTLA4 los resultados obtenidos en carcinoma renal fueron que se encontró una asociación entre los polimorfismos del gen del CTLA-4 y un incremento del riesgo de desarrollar RCC. Se descubrió una alta frecuencia genotípica de los polimorfismos CTLA4/CT60-AA y CTLA4/A49G-AA en pacientes de RCC frente a los controles. Se descubrió una asociación entre el polimorfismo CTLA4/CT60 y el grado tumoral en pacientes de RCC, un análisis logístico de la regresión confirmó los hallazgos, demostrando una alta frecuencia del genotipo AA en pacientes con grado tumoral alto.

La conclusión apoya la hipótesis de que diversos factores genéticos implicados en la regulación de respuestas inmunes adaptativas, la composición de las células estromales y el nivel de producción local de citoquinas, pueden ser cruciales en la modificación de parámetros clinicopatológicos en el carcinoma renal.

Palabras clave: Cáncer renal. Polimorfismos genéticos. Respuesta inflamatoria.

\section{Polymorphisms in inflammatory response genes in metastatic renal cancer Abstract}

Inflammation has been implicated as an etiological factor in different human cancers. Allelic variations in the genes implicated in inflammation are candidates as genetic determinants or markers of renal carcinoma risk. The present study investigates whether polymorphisms of the genes that give rise to increases in the levels of proinflammatory cytokines and chemokines are associated with an increased risk of renal carcinoma. To this effect, a number of case-control studies were designed to assess the correlation between renal carcinoma and polymorphisms IL10-1082 A/G (rs 1800896), IL10-592 A/C (rs 1800872), IL10-819 C/T (rs 1800871), IL10-1082 A/G, IL4-590 C/T (rs 2243250), TNF-A-308 A/G (rs 1800629), RANTES-403 G/A (rs 2107538), IL1-A-889 C/T (rs 1800587), MCP-1 2518 G/A (rs 1024611), CTLA-4/+49 A/G (rs 231775) and CTLA-4 CT60 A/G (rs 3087243) in 127 renal carcinoma patients and in 176 healthy subjects.

The results obtained in relation to cytokine polymorphism IL-10-1082 A/G indicate that AG heterozygosity status is the principal risk factor in relation to locally advanced or metastatic tumor stage and renal carcinoma. In the case of the molecule CTLA4, the results obtained in renal cancer reveal an association between the polymorphisms of the CTLA-4 gene and an increased risk of developing renal cell carcinoma. A high genotypic frequency of polymorphisms CTLA4/CT60-AA and CTLA4/A49G-AA is observed in patients with renal cell carcinoma versus the controls. An association has been established between polymorphism CTLA4/CT60 and tumor grade in patients with renal cell carcinoma. Logistic regression analysis has confirmed these data, demonstrating a high frequency of the AA genotype in patients with high-grade tumors.

The results obtained support the hypothesis that different genetic factors implicated in the regulation of adaptive immune responses, stromal cell composition and local cytokine production levels may be crucial elements in the modification of the clinicopathological parameters of renal carcinoma.

Keywords: Renal cancer. Genetic polymorphisms. Inflammatory response. 
$\mathrm{L}^{\mathrm{a}}$ a inflamación crónica de larga duración ha sido relacionada con el desarrollo de varios tipos de cáncer. El mecanismo de carcinogénesis involucraría el daño repetitivo y la generación y aparición en los tejidos de especies altamente reactivas del oxígeno y de nitrógeno. Estas moléculas reactivas, como el óxido nítrico y $\mathrm{H}_{2} \mathrm{O}_{2}$ serían liberadas por las células inflamatorias y interaccionarían con el ADN del epitelio en proliferación y podrían producir de esta manera alteraciones genéticas. Diversos estudios epidemiológicos han sugerido una fuerte asociación entre la infección crónica, la inflamación y el cáncer $^{1-6}$. Por ejemplo, hay una fuerte asociación entre el abuso de alcohol, con la inflamación del hígado y páncreas, y la exposición y consumo de tabaco con procesos inflamatorios y riesgo de padecer cáncer de pulmón.

Otros ejemplos lo constituyen, la enfermedad inflamatoria intestinal y el cáncer de colon, la infección por Helicobacter pylori y el cáncer gástrico, la infección por virus de hepatitis y cáncer hepático, la infección por Schistosoma y el cáncer de vejiga, del virus del papiloma con el cáncer cervical y del virus EBV con el cáncer nasofaríngeo y el linfoma de Burkitt. Todas estas asociaciones sugieren que la inflamación crónica puede estar involucrada en el inicio de la transformación neoplásica (el proceso en el que las células son alteradas genéticamente), promoción (el proceso en el que un grupo de células son estímuladas para proliferar) y la progresión (el proceso mediante el cual adquieren un comportamiento más agresivo e invasivo). Existen por tanto, numerosas evidencias que apoyan que si bien la carcinogénesis es un proceso dependiente de la acumulación de cambios genéticos o epigenéticos en las células transformadas (factores intrínsecos), muchas etapas son necesarias para la progresión tumoral; por ejemplo, proliferación, invasión angiogénesis, son influenciadas por factores microambientales (extrínsecos) tales como factores de crecimiento, factores angiogénicos, citoquinas y enzimas proteolíticas ${ }^{7}$.

En adición a los datos epidemiológicos que sostienen una estrecha relación entre inflamación crónica y cáncer ${ }^{1,2,8}$, ciertos polimorfismos genéticos, situados en los promotores de citoquinas de la inmunidad natural, están asociados a incremento del riesgo de desarrollar cáncer.

Mutaciones y/o polimorfismos genéticos en genes cruciales que regulan funciones clave del sis- tema inmune y la supervivencia de los linfocitos han sido implicados como factores etiológicos en la inflamación crónica ${ }^{9,10}$

En resumen, en el microambiente tumoral, va a existir un delicado balance entre factores que promueven el desarrollo de la respuesta inflamatoria, favorecedores del desarrollo neoplásico, y factores inhibidores, también producidos por células de la inmunidad innata o específica y por células estromales que tienen efectos contrapuestos, inhibiendo el proceso inflamatorio y favoreciendo el proceso apoptótico. Este balance puede entonces verse notablemente influenciado por factores genéticos (i.e., polimorfismos genéticos), que predisponen al desarrollo de respuestas inflamatorias.

El carcinoma celular renal (RCC) se caracteriza por ser un cáncer muy angiogénico y por haber una alta infiltración linfocitaria y producción de citoquinas ${ }^{11}$ y representa un $3-6 \%$ de todos los tumores adultos ${ }^{12}$. La inmunoterapia con IFN- $\alpha$ o IL-2 es una manera efectiva para tratar los pacientes metastáticos de cáncer renal. El CCR muestra una buena respuesta a inmunoterapia destinada a bloquear la actividad del CTLA- $4^{13}$, esto nos indica el rol importante del sistema inmune en la regulación del crecimiento tumoral del CCR.

Hemos realizado diferentes estudios de polimorfismo genéticos relacionados con la producción de citoquinas y quimioquinas proinflamatorias en el carcinoma renal. Los genes de las citoquinas y quimioquinas estudiadas han sido la IL-10, IL-4, TNF$\alpha$, MCP- 1 , IL- $1 \alpha$, RANTES y el gen CTLA- 4 cuyo producto regula la intensidad y duración de las respuestas inmunológicas adaptativas mediadas por los linfocitos T. La elección de estas citoquinas y quimioquinas para los estudios es debido a su implicación en las respuestas de estrés celular, inflamación y persistencia de la respuesta inmunológica.

\section{MATERIAL Y MÉTODOS}

El estudio incluía 127 pacientes de RCC, y como control 176 personas sanas. Se extrajo el DNA de las sangre periférica de estos pacientes y controles utilizando el QIAamp DNA Mini kit. y se realizó el genotipado de los polimorfismos de nucleótidos (SNP) por medio de un ensayo alélico discriminatorio utilizando la TaqMan 5 ${ }^{\circ}$. La PCR y el genotipado de cada muestra fue directamente atribuible mediante la medición de la fluorescencia alélica 
específica en la 7500 PCR-REAL TIME Sequence Detection Systems utilizando el software SDS 2.2.1 para la discriminación alélica (Applied Biosystems, Foster City, CA, USA). Los ensayos de amplificación de los SNPs se realizaron según el protocolo siguiente: 10 ng de la muestra $\mathrm{ADN}(1 \mu \mathrm{L})$ fue mezclado con 4 $\mu \mathrm{L}$ de solución de reacción que contiene: $2,5 \mu \mathrm{L}$ de $2 \mathrm{X}$ TaqMan® Universal PCR Mix (Applied Biosystems), $0,25 \mu \mathrm{l}$ de una mezcla de dos primers y dos sondas MGB-Taqman(20X) (Applied Biosystems), y 1,25 $\mu \mathrm{L}$ de agua destilada. las condiciones de la reacción de la PCR fueron: preincubación a $50^{\circ} \mathrm{C}$ durante $2 \mathrm{~min}$ y a $95^{\circ} \mathrm{C}$ durante $10 \mathrm{~min}$, seguido de 40 ciclos a $95^{\circ} \mathrm{C}$, $15 \mathrm{~s} ; 60^{\circ} \mathrm{C}, 1 \mathrm{~min}$.

Los polimorfismos de las citoquinas-quimioquinas y moléculas estudiados se encuentran en la región promotora y afectan a los niveles de trascripción. Los polimorfismos estudiados son: IL10-1082 A/G (rs 1800896), IL10-592 A/C (rs 1800872), IL10-819 C/T (rs 1800871), IL10-1082 A/G, IL4-590 C/T (rs 2243250), TNF-A -308 A/G (rs 1800629), RANTES 403 G/A (rs 2107538), IL1-A -889 C/T (rs 1800587), MCP-1 2518 G/A (rs 1024611) y CTLA-4/ +49 A/G (rs 231775) y CTLA-4 CT60 A/G (rs 3087243).

En la Figura 1 se muestra el resultado de la tipificación del polimorfismo genético de IL-10-1082 mediante la tecnología Taqman en un 7500 PCRREAL TIME Sequence Detection Systems.

\section{ANÁLISIS ESTADÍSTICO}

Los datos fueron compilados de acuerdo al genotipo y la frecuencia alélica estimada de lo observado en el número de cada alelo especifico. La frecuencia alélica de los polimorfismos de un unico nucleotido fue testado contra el equilibrio de Hardy-Weinberg

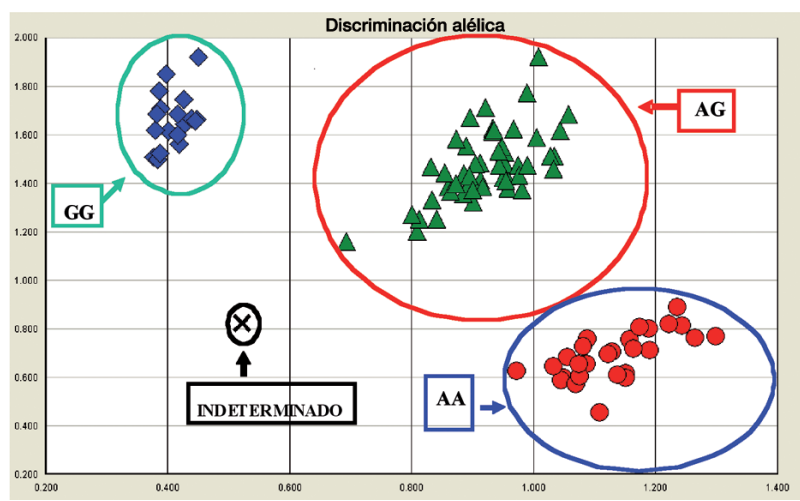

FIGURA 1. Resultado de la discriminación alélica obtenido en el analizador 7500 PCR-REAL TIME Sequence Detection Systems en muestras para estudio del polimorfismo de IL-10-1082 A/G. mediante la comparación observada y la frecuencia genotípica esperada utilizando el test de la $\chi^{2}$. Las frecuencias genotípicas fueron comparadas en tablas de $2 \times 2$ con el test de $\chi^{2}$ y la corrección de Yates o con el test exacto de Fisher cuando el valor esperado es <5. La fuerte asociación fue estimada mediante el calculo de la odds ratios (ORs) con un intervalo de confianza (CI) del 95\% y un P valor. Los genotipos fueron comparados con una serie de datos clínicos como el estadio o el tamaño del tumor. El análisis de la regresión logística se utilizó para analizar la interacción entre SNPs y para confirmar en las frecuencias de los polimorfismos entre casos y controles. El software SPSS v.15.0 se utilizó para el análisis estadístico.

\section{RESULTADOS Y DISCUSIÓN \\ Polimorfismos genéticos de citoquinas y genes de la inmunidad adaptativa}

IL-10 es una potente citoquina con efectos pleitrópicos y puede actuar como un agente que promueve el cáncer ${ }^{14}$. Desde siempre a la IL-10 se la ha considerado una potente citoquina TH2, con actividad inmunosupresiva y antiinflamatoria, mediante la inhibición de la replicación de linfocitos y monocitos y la secreción de citoquinas inflamatorias. No se sabe muy bien el significado de la producción de la IL-10 en el desarrollo tumoral y su microambiente. Se han encontrado descubrimientos opuestos cuando se han medido los niveles de IL-10 en sangre periférica (altos niveles se relacionaba con un peor pronostico tumoral) o en las muestras tumorales (altos niveles estaba relacionado con supervivencia) ${ }^{15}$.

Los resultados observados de los polimorfismos IL10-1082 A/G, IL10-592 A/C, IL10-819 C/T (estos dos últimos se encuentran en desequilibrio de unión con el IL-10-1082) en carcinoma renal fueron los siguientes: No hemos encontrado asociación en pacientes de RCC entre el estudio de polimorfismos y el estado tumoral (TNM) o la edad y el género del paciente. No ha habido diferencias significativas en los polimorfismos de los promotores de la IL-10 entre pacientes de RCC y controles. En relación con el diámetro del tumor un incremento en el genotipo AG se obtuvo en pacientes con tumores mayores de $7 \mathrm{~cm}$ el OR para la heterocigosidad era de 4,41 ( $\mathrm{p}=0,001)$ (Tabla 1). El análisis estadístico demostró que el alelo $\mathrm{G}$ resulto ser un alelo de riesgo $(\mathrm{OR}=3,66, \mathrm{p}=0,003)$, (Tabla 1). No se encontraron diferencias en el grado 
Tabla 1. Riesgo genotípico y significación estadística del polimorfismo IL-10 1082 SNP en parámetros clínicos del carcinoma renal

\begin{tabular}{|c|c|c|c|c|c|}
\hline Parámetros clínicos & Alelo & Frecuencia alélica & Heterocigosidad & Homocigosidad & Positivad alélica \\
\hline $\begin{array}{l}\text { Diámetro tumoral } \\
\text { mayor de } 7 \mathrm{~cm}\end{array}$ & $\begin{array}{l}\text { Alelo A } \\
\text { Alelo G }\end{array}$ & $\begin{array}{l}\mathrm{OR}=0,62 \\
\mathrm{p}=0,084 \\
\mathrm{OR}=1,61 \\
\mathrm{p}=0,084\end{array}$ & $\begin{array}{l}\mathrm{OR}=2,36 \\
\mathrm{p}=0,133 \\
\mathbf{O R}=\mathbf{4 , 4 1} \\
\mathbf{p}=\mathbf{0 , 0 0 1}\end{array}$ & $\begin{array}{l}\mathrm{OR}=0,54 \\
\mathrm{p}=0,328 \\
\mathrm{OR}=1,87 \\
\mathrm{p}=0,328\end{array}$ & $\begin{array}{l}\mathrm{OR}=1,38 \\
\mathrm{p}=0,563 \\
\mathbf{O R}=\mathbf{3}, \mathbf{6 6} \\
\mathbf{p}=\mathbf{0 , 0 0 3}\end{array}$ \\
\hline Grado nuclear G3-4 & $\begin{array}{l}\text { Alelo A } \\
\text { Alelo G }\end{array}$ & $\begin{array}{l}\mathrm{OR}=1,04 \\
\mathrm{p}=0,877 \\
\mathrm{OR}=0,96 \\
\mathrm{p}=0,877\end{array}$ & $\begin{array}{l}\mathrm{OR}=1,16 \\
\mathrm{p}=0,809 \\
\mathrm{OR}=1,01 \\
\mathrm{p}=0,978\end{array}$ & $\begin{array}{l}\mathrm{OR}=1,14 \\
\mathrm{p}=0,833 \\
\mathrm{OR}=0,87 \\
\mathrm{p}=0,833\end{array}$ & $\begin{array}{l}\mathrm{OR}=1,15 \\
\mathrm{p}=0,807 \\
\mathrm{OR}=0,98 \\
\mathrm{p}=0,964\end{array}$ \\
\hline Adenopatías (pN+) & $\begin{array}{l}\text { Alelo A } \\
\text { Alelo G }\end{array}$ & $\begin{array}{l}\mathrm{OR}=0,65 \\
\mathrm{p}=0,348 \\
\mathrm{OR}=1,55 \\
\mathrm{p}=0,348\end{array}$ & $\begin{array}{l}\mathrm{OR}=0,56 \\
\mathrm{p}=0,074 \\
\mathbf{O R}=\mathbf{1 , 7 9} \\
\mathbf{p}=\mathbf{0 , 0 0 6}\end{array}$ & $\begin{array}{l}\mathrm{OR}=0,43 \\
\mathrm{p}=1.000 \\
\mathrm{OR}=2,33 \\
\mathrm{p}=1,000\end{array}$ & $\begin{array}{l}\mathrm{OR}=0,63 \\
\mathrm{p}=0,176 \\
\mathrm{OR}=1,59 \\
\mathrm{p}=0,015\end{array}$ \\
\hline Trombosis venosa & $\begin{array}{l}\text { Alelo A } \\
\text { Alelo G }\end{array}$ & $\begin{array}{l}\text { OR }=1,13 \\
p=0,776 \\
\text { OR=0,88 } \\
p=0,776\end{array}$ & $\begin{array}{l}\mathrm{OR}=0,84 \\
\mathrm{p}=0,210 \\
\mathrm{OR}=2,14 \\
\mathrm{p}=0,269\end{array}$ & $\begin{array}{l}\mathrm{OR}=0,69 \\
\mathrm{p}=0,547 \\
\mathrm{OR}=1,46 \\
\mathrm{p}=0,547\end{array}$ & $\begin{array}{l}\mathrm{OR}=0,84 \\
\mathrm{p}=0,210 \\
\mathrm{OR}=1,62 \\
\mathrm{p}=0,489\end{array}$ \\
\hline Metástasis (M1) & $\begin{array}{l}\text { Alelo A } \\
\text { Alelo G }\end{array}$ & $\begin{array}{l}\text { OR }=1,04 \\
p=0,923 \\
\text { OR=0,96 } \\
p=0,923\end{array}$ & $\begin{array}{l}\mathrm{OR}=0,80 \\
\mathrm{p}=0,756 \\
\mathrm{OR}=0,81 \\
\mathrm{p}=0,709\end{array}$ & $\begin{array}{c}\text { OR }=0,98 \\
p=0,97 \\
\text { OR }=1,02 \\
p=0,98\end{array}$ & $\begin{array}{c}\mathrm{OR}=0,87 \\
\mathrm{p}=0,84 \\
\mathrm{OR}=0,86 \\
\mathrm{p}=0,77\end{array}$ \\
\hline $\begin{array}{l}\text { Estadío tumoral } \\
\text { localmente avanzado } \\
\text { o metastásico }\end{array}$ & $\begin{array}{l}\text { Alelo A } \\
\text { Alelo G }\end{array}$ & $\begin{array}{l}\mathrm{OR}=0,62 \\
\mathrm{p}=0,189 \\
\mathrm{OR}=1,61 \\
\mathrm{p}=0,189\end{array}$ & $\begin{array}{c}\mathrm{OR}=0,78 \\
\mathrm{p}=0,097 \\
\mathbf{O R}=\mathbf{1 4 , 1 0} \\
\mathbf{p}=\mathbf{0 , 0 0 2}\end{array}$ & $\begin{array}{l}\mathrm{OR}=0,40 \\
\mathrm{p}=0,509 \\
\mathrm{OR}=2,48 \\
\mathrm{p}=0,509\end{array}$ & $\begin{array}{c}\mathrm{OR}=0,31 \\
\mathrm{p}=0,462 \\
\mathbf{O R}=\mathbf{1 1} \mathbf{1} \mathbf{0 4} \\
\mathbf{p}=\mathbf{0 , 0 0 5}\end{array}$ \\
\hline Riesgo UCLA medio o alto & Alelo G & $\begin{array}{l}\mathrm{OR}=0,60 \\
\mathrm{p}=0,097 \\
\mathrm{OR}=1,67 \\
\mathrm{p}=0,097\end{array}$ & $\begin{array}{l}\mathrm{OR}=0,59 \\
\mathrm{p}=0,462 \\
\mathrm{OR}=1,90 \\
\mathrm{p}=0,167\end{array}$ & $\begin{array}{l}\mathrm{OR}=3,21 \\
\mathrm{p}=0,109 \\
\mathrm{OR}=0,31 \\
\mathrm{p}=0,109\end{array}$ & $\begin{array}{c}\mathrm{OR}=0,47 \\
\mathrm{p}=0,4260 \\
\mathrm{OR}=2,10 \\
\mathrm{p}=0,098\end{array}$ \\
\hline
\end{tabular}

${ }^{1}$ Calculado mediante comparación de heterocigosidad vs homocigosidad sin el test de alelo

${ }^{2}$ Calculado mediante comparación de homocigosidad con la prueba alélica VS homocigosidad

nuclear, en la aparición de trombosis maligna, aparición de metástasis o riesgo UCLA para el polimorfismo. En el caso de riesgo UCLA, la combinación bajamedio contra alto riesgo se analizo y no se encontró ninguna relación. Los pacientes de carcinoma renal que presentaban adenopatía se vio que todos ellos presentaban heterocigosidad (AG); el análisis estadístico de Fisher reporto significación, con un OR de 1,79 ( $p=0,006$ ) (Tabla 1). Otro parámetro estudiado fue el estadio tumoral, que relaciona el diámetro tumoral con la adenopatía, dándonos el más alto el genotipo heterocigoto $(\mathrm{OR}=14,10 ; \mathrm{p}=0,002)$ (Tabla 1). Estos resultados están en concordancia con los obtenidos en el diámetro tumoral. Por lo tanto el estado de heterocigosidad AG presenta el factor de riesgo principal en relación con estadio tumoral local avanzado o más avanzado. Los resultados del análisis de haplotipos no nos reportó ninguna información adicional en comparación con el análisis independiente del polimorfismo - 1082 .

Para concluir el trabajo realizado en RCC sobre los polimorfismos de la IL-10 nosotros pensamos que una alta producción de IL-10, puede a la vez producir un efecto imunosupresor sobre los mecanismos de inmunidad antitumoral, pero también de forma paralela producir un efecto inhibitorio sobre el crecimiento tumoral al activarse mecanismos anti-angiogénicos que primarían sobre los primeros. Por el contrario, en un entorno de baja producción de IL-10, se podría facilitar respuestas antitumorales más potentes. Sería por tanto en individuos con un fenotipo intermedio de producción de IL-10 en donde se encontraría la situación más favorable para la progresión tumoral. En este contexto el esta- 
tus de heterocigosidad (la producción intermedia de IL-10) puede producir suficientes efectos inmunosupresivos mediante la inhibición del linfocito $\mathrm{T}$ helper 1(TH1) proinflamatorio y la respuesta citotóxica, y al mismo tiempo no induce un potente efecto antiangiogenico favoreciendo la progresión tumoral en el carcinoma renal celular.

Nosotros creemos que la composición de las células estromales y el nivel de producción local pueden ser cruciales para la observación de un tipo $\mathrm{u}$ otro de respuesta y explicar así los aparentemente resultados contradictorios, encontrados en distintos tipos de tumores.

IL-4 es una citoquina producida por las células T de tipo 2 (Th2), basofilos, mastocitos y eosinofilos activados. Actúa como componente antiinflamatorio al bloquear la síntesis de IL-1, TNF- $\alpha$, IL-6 y la proteina inflamatoria del macrofago. Participa en la regulación del sistema inmunológico en múltiple niveles. Entre otras funciones, promueve la diferenciación de linfocitos Th2, la proliferación y diferenciación de linfocitos $\mathrm{B}$ y es un potente inhibidor de la apoptosis. En el estudio realizado sobre el polimorfismo IL4-590 C/T en RCC y controles no hemos encontrado resultados significativos en el estado tumoral la edad ni el genero del paciente. Tampoco encontramos diferencias significativas entre pacientes y controles.

CTLA-4 es una molécula que regula la intensidad y duración de la respuesta inmune adaptativa mediada por linfocitos T. CTLA-4 juega un papel importante en la regulación negativa de la proliferación y activación de los linfocitos $\mathrm{T}$. La molécula CTLA-4 interacciona con el ligando B7 en la superficie de las células presentadoras de antígenos, provocando una parada del ciclo celular del linfocito $\mathrm{T}$ y una inhibición de la producción de citoquinas y por consiguiente elimina la fase de proliferación de linfocitos $\mathrm{T}$ que es fundamental como respuesta antitumoral.

Se encontró una asociación entre los polimorfismos del gen del CTLA-4 y un incremento del riesgo de desarrollar RCC. Se descubrió una alta frecuencia genotípica de los polimorfismos CTLA4/CT60-AA y CTLA4/A49G-AA en pacientes de RCC frente a los controles (CTLA4/CT60-AA: 38,6\% VS 22,9\% en controles, $\mathrm{p}=0,005$ y un $\mathrm{OR}=2,12$ con $95 \% \mathrm{CI}$ : 1,28-3,50; y para CLA4/A49G-AA: 58,4\% frente a 44,3\% en controles, $\mathrm{P}=0,022$ y $\mathrm{OR}=1,76$ con $95 \% \mathrm{CI}$ : $1,11-2,80$ ) (Tabla 2). Un análisis de regresión multivariante no produjo ninguna información adicional, aunque un alto OR se obtuvo para CTLA-4/CT60 (OR=2,59 con 95\% CI: 1,40-5,3). La distribución alélica en los controles fue similar a los publicados en estudios previos en pacientes españoles ${ }^{32}$. Se descubrió una asociación entre el polimorfismo CTLA-4/CT60 y el grado tumoral en pacientes de RCC (Tabla 3). El análisis logístico de la regresión confirmó los hallazgos, demostrando una alta frecuencia del genotipo AA en pacientes de alto grado.

No hemos encontrado asociación en pacientes de RCC entre el estudio de polimorfismos y el estado tumoral (TNM) o la edad y el género del paciente.

Como conclusiones al estudio de los polimorfismos de la molécula de CTLA-4 en pacientes de RCC hay que destacar el incremento de la frecuencia genotípica de CTLA-4/CT60-AA y CTLA-4/A49G-AA en RCC, que nos indica la asociación del polimorfismos de CTLA-4 con el incremento del riesgo de desarrollar RCC. El polimorfismo CTLA-4/CT60 se cree que controla el procesamiento y la producción de CTLA-4. Se ha encontrado que la varianza alélica CTLA-4/CT60 G esta correlacionado con una reducción de los niveles de ARNm de la forma alternativa de pliegue de CTLA-4, la cual inhibe la proliferación in vitro de células $\mathrm{T}^{33}$. Al encontrarnos una frecuencia mayor de CTLA-4/CT60-AA, la proliferación de los linfocitos $\mathrm{T}$ disminuiría facilitando el desarrollo del tumor renal. Lo mismo ocurre con el CTLA-4/ A49G donde en individuos con genotipo $+49 \mathrm{G}$ se ha visto que se produce un aumento en la proliferación linfocitaria en condiciones de activación suboptimas $^{34}$, mientras que en los pacientes de RCC hay un incremento de la frecuencia genotípica de CTLA4/A49G-AA, con lo que presumiblemente no habría una proliferación linfocitaria en condiciones de activación subóptimas.

\section{Polimorfismos genéticos de citoquinas de la respuesta inmune innata}

TNF-A es un mediador clave de la inflamación y pude contribuir a la iniciación del tumor mediante la estimulación de la producción de moléculas genotóxicas, que pueden llevar a un daño en el ADN y mutaciones ${ }^{16}$. Variaciones interindividuales en los niveles de TNF- $\alpha$ se han atribuido a los polimorfismos, notablemente a la posición -380(G/A) en la región promotora del gen TNF- $\alpha$, y el alelo $\mathrm{A}$ se ha asociado con altos niveles de trascripción del TNF-A ${ }^{17} \mathrm{y}$ al incremento del riesgo para varios tipos de cáncer ${ }^{18}$. 
Tabla 2. Análisis estadístico de polimorfismos en RCC VS grupo control

\begin{tabular}{|c|c|c|c|c|c|}
\hline Genotipo & RCC Pacientes & $(n=127)$ & Controles $(n=176)$ & OR $(95 \% \mathrm{CI})$ & P-Valor \\
\hline \multirow{3}{*}{ IL-10 1082} & AA & 42 (33,3\%) & $58(33,1 \%)$ & $1,01(0,62-1,64)$ & 1,02 \\
\hline & AG & $62(49,2 \%)$ & $87(49,7 \%)$ & $1,02(0,65-1,61)$ & 1,00 \\
\hline & GG & $22(17,5 \%)$ & $30(17,1 \%)$ & $0,98(0,53-1,79)$ & 1,01 \\
\hline \multirow[t]{3}{*}{ IL-10 819 (592) } & $\mathrm{CC}(\mathrm{CC})$ & $81(63,8 \%)$ & 98 (56\%) & $1,38(0,87-2,21)$ & 0,21 \\
\hline & $\mathrm{CT}(\mathrm{AC})$ & $37(29,1 \%)$ & $63(36 \%)$ & $1,37(0,84-2,24)$ & 0,26 \\
\hline & TT (AA) & $9(7,1 \%)$ & $14(8 \%)$ & $1,14(0,48-2,72)$ & 0,94 \\
\hline \multirow[t]{6}{*}{ IL-10 Combinado } & $\mathrm{ACC} / \mathrm{ACC}$ & $14(11,1 \%)$ & $19(10,9 \%)$ & $1,02(0,49-2,12)$ & 1,00 \\
\hline & ACC/ATA & $19(15,1 \%)$ & $25(14,4 \%)$ & $1,06(0,56-2,02)$ & 0,99 \\
\hline & $\mathrm{ACC} / \mathrm{GCC}$ & 44 (34,9\%) & $49(28,2 \%)$ & $1,37(0,84-2,24)$ & 0,26 \\
\hline & ATA/ATA & $9(7,1 \%)$ & $14(8 \%)$ & $0,88(0,37-2,10)$ & 0,94 \\
\hline & ATA/GCC & $18(14,3 \%)$ & $37(21,3 \%)$ & $0,62(0,33-1,14)$ & 0,16 \\
\hline & $\mathrm{GCC} / \mathrm{GCC}$ & $22(17,5 \%)$ & $30(17,2 \%)$ & $1,02(0,55-1,86)$ & 1,00 \\
\hline \multirow[t]{3}{*}{ IL-4 } & $\mathrm{CC}$ & $93(73,2 \%)$ & $123(70,7 \%)$ & $1,13(0,68-1,89)$ & 0,72 \\
\hline & $\mathrm{CT}$ & $30(23,6 \%)$ & $47(27 \%)$ & $1,20(0,70-2,03)$ & 0,59 \\
\hline & $\mathrm{TT}$ & $4(3,2 \%)$ & $4(2,3 \%)$ & $0,72(0,18-2,95)$ & $0,73^{*}$ \\
\hline \multirow[t]{3}{*}{ CTLA4 /CT60 } & AA & $49(38,6 \%)$ & $40(22,9 \%)$ & $2,12(1,28-3,50)$ & $0,005^{\#}$ \\
\hline & AG & $55(43,3 \%)$ & $88(50,3 \%)$ & $1,32(0,84-2,10)$ & 0,28 \\
\hline & GG & $23(18,1 \%)$ & 47 (26,9\%) & $1,66(0,95-2,91)$ & 0,10 \\
\hline \multirow[t]{3}{*}{ CTLA4 /A49G } & AA & $73(58,4 \%)$ & $78(44,3 \%)$ & $1,76(1,11-2,80)$ & $\mathbf{0 , 0 2 2} 2^{\#}$ \\
\hline & AG & $43(34,4 \%)$ & 77 (43,8\%) & $1,48(0,92-2,38)$ & 0,13 \\
\hline & GG & $9(7,2 \%)$ & $21(11,9 \%)$ & $1,74(0,77-3,95)$ & 0,25 \\
\hline
\end{tabular}

Abreviaturas: RCC, cáncer renal; OR, rango de riesgo; CI, intervalo de confianza

*Fisher test

\#Diferencias estadísticas significativas entre pacientes con carcinoma renal y controles,

En un estudio previo realizado en tejido de carcinoma de próstata comparando el polimorfismo del promotor del TNF- $\alpha$ reveló diferencias estadísticas significativas en la distribución del genotipo para el locus -308, con una alta frecuencia del alelo A (AA/AG) en pacientes con controles $(\mathrm{OR}=1,62 ; 95 \%$ CI:1.077$2.436 \mathrm{p}=0,020$ ). No se encontraron relaciones con parámetros clinicopatológicos y este polimorfismo de promotor de TNF-A. El estudio realizado en carcinoma renal en contraste no reveló asociación con riesgo y progresión en el cáncer renal metastásico.

IL-1A es una citoquina proinflamatoria producida por monocitos, macrófagos, y células epiteliales y tiene características biológicas similares al TNF-A, incluyendo la participación en la respuesta a la invasión microbial, inflamación y daño tisular ${ }^{22}$. Los polimorfismos de la IL-1 se ha asociado a cáncer gástrico $^{23}$ hepatocelular ${ }^{24}$, pulmón ${ }^{25}$, colon ${ }^{26}$, vulva $^{26}$ y ovario ${ }^{27}$. El polimorfismo $\mathrm{C} / \mathrm{T}$ en la región -889 del promotor de la IL1-A fue estudiado; el alelo $\mathrm{T}$ causa una alta expresión de la citoquina. El estudio de este polimorfismo no reveló asociación con riesgo y progresión del cáncer renal y prostático.

\section{Polimorfismos genéticos de quimiocinas}

RANTES es una quimioquina que normalmente es expresada y presumiblemente secretada por las células T. Se cree que juega un rol importante en la inmunidad antitumoral mediante el reclutamiento de células de la inmunidad ${ }^{19}$. Polimorfismos en RANTES se han asociado con un alto riesgo de cáncer pancreático apoyado por la hipótesis de que los polimorfismos de genes proinflamatorios en combinación con condiciones proinflamatorias puede influir en el desarrollo del cáncer de páncreas ${ }^{20}$. Los polimorfismos estudiados A/G se encuentran en la región del promotor -403; el alelo A está asociado a una alta trascripción del gen ${ }^{21}$.

En el trabajo previo, realizado en cáncer de próstata, los pacientes mostraron una alta frecuencia del alelo A en el polimorfismo de RANTES -403 G/A frente a controles $(\mathrm{OR}=1.807$; 95\% CI: 1.149-2.844 $\mathrm{p}=0,014)$. Sin embargo como ocurrió con TNFA, en el cáncer renal no se encontró asociación con parámetros clinicopatológicos.

MCP-1 es un miembro de la familia de las CC quimioquinas. Posee actividad quimiotactico, para monocitos y linfocitos $\mathrm{T}^{28,29}$ y se cree que juega un 
Tabla 3. Asociación del estudio de polimorfismos y el grado clínico y estadio en RCC

\begin{tabular}{|c|c|c|c|c|c|c|c|c|c|}
\hline Genotipo & & $\begin{array}{c}\text { Bajo } \\
\text { (T1-NOM0) }\end{array}$ & $\begin{array}{c}\text { Medio } \\
\text { (T3-4NOMO) }\end{array}$ & $\begin{array}{c}\text { Alto } \\
(\mathbf{T x}, \mathbf{N}+\mathbf{M}+)\end{array}$ & $\mathbf{x}^{2}$ & G1 & G2 & G3 & P-Valor \\
\hline \multirow{3}{*}{ IL-10 1082} & AA & 31 & 7 & 3 & No & 10 & 18 & 12 & \\
\hline & $\mathrm{AG}$ & 37 & 7 & 17 & posible de & 17 & 22 & 21 & $\mathrm{P}=0,74$ \\
\hline & GG & 17 & 3 & 1 & calcular & & 3 & 9 & 8 \\
\hline \multirow[t]{3}{*}{ IL 10-819 (592) } & $\mathrm{CC}(\mathrm{CC})$ & 58 & 8 & 14 & & 18 & 35 & 26 & \\
\hline & $\mathrm{CT}(\mathrm{AC})$ & 22 & 7 & 6 & $P=0,59$ & 10 & 10 & 13 & $\mathrm{P}=0,73$ \\
\hline & TT (AA) & 6 & 2 & 1 & & 2 & 4 & 3 & \\
\hline \multirow[t]{3}{*}{ IL-4 } & $\mathrm{CC}$ & 59 & 13 & 18 & & 24 & 30 & 34 & \\
\hline & $\mathrm{CT}$ & 24 & 3 & 3 & $P=0,49$ & 6 & 17 & 7 & $P=0,19$ \\
\hline & $\mathrm{TT}$ & 3 & 1 & 0 & & 0 & 2 & 1 & \\
\hline \multirow[t]{3}{*}{ CTLA4 /CT60 } & $\mathbf{A A}$ & 34 & 5 & 9 & & 8 & 15 & 25 & \\
\hline & $\mathrm{AG}$ & 39 & 8 & 8 & $\mathrm{P}=0,85$ & 17 & 24 & 11 & $\mathrm{p}=0,022^{*}$ \\
\hline & GG & 13 & 4 & 4 & & 5 & 10 & 6 & \\
\hline \multirow[t]{3}{*}{ CTLA4 /A49G } & AA & 50 & 11 & 11 & & 19 & 27 & 25 & \\
\hline & $\mathrm{AG}$ & 33 & 4 & 6 & $\mathrm{P}=0,48$ & 10 & 20 & 11 & $\mathrm{p}=0,54$ \\
\hline & GG & 3 & 2 & 2 & & 1 & 2 & 4 & \\
\hline
\end{tabular}

*Diferencias estadísticas significativas con un intervalo de confianza de 95\%

rol fundamental en el reclutamiento de macrófagos, expresión de factores angiogénicos y activación de la matriz metaloproteinasa en pacientes de cáncer de mama. Variaciones genéticas dentro de la región reguladora que afecta a la trascripción y producción de la proteína MCP-1 se ha correlacionado con el riesgo de metástasis en el cáncer de mama ${ }^{30}$. Estos descubrimientos se correlacionan con la sobreexpresión de MCP-1 en tejido tumoral de mama de un estudio anterior ${ }^{31}$. En el estudio realizado en tejido cáncer renal y prostático no reveló resultados significativos.

En resumen, diversos factores genéticos implicados en la regulación de respuestas inmunes adaptativas (IL-10 y CTL-A4) se encuentran asociados a parámetros clinicopatológicos y riesgo en el carcinoma renal de células claras. En contra de lo que sucede en el cáncer de próstata, el cáncer renal no parece asociarse a polimorfismos genéticos que regulan la respuesta inmunes innata. No obstante estudios sobre series más largas son necesarios para confirmar nuestros resultados.

\section{REFERENCIAS}

1. Coussens LM, Werb Z Inflammation and cancer. Nature. 2002 Dec 19-26;420(6917):860-867.

2. Shacter E, Weitzman SA. Chronic inflamation and cancer. Oncology (Williston Park). 2002 Feb;16(2):217-26, 229; discussion 230-232.

3. Karin M, Cao Y, Greten FR, Li ZW. NF-kappaB in cancer: from innocent bystander to major culprit..Nat Rev Cancer. 2002 Apr;2(4):301-310.
4. de Visser KE, Eichten A, Coussens LM. Paradoxical roles of the immune system during cancer development. Nat Rev Cancer. 2006 Jan;6(1):24-37.

5. Balkwill F, Charles KA, Mantovani A. Smoldering and polarized inflammation in the initiation and promotion of malignant disease. Cancer Cell. 2005,7(3):211-217.

6. Lin WW, Karin M. A cytokine-mediated link between innate immunity, inflammation, and cancer. J Clin Invest. 2007;117 (5): 1175-1183.

7. Zitvogel L, Tesniere A, Kroemer G. Cancer despite immunosurveillance: immunoselection and immunosubversion. Nat Rev Immunol. 2006 Oct;6(10):715-727.

8. Luo JL, Maeda S, Hsu LC, Yagita H, Karin M. Inhibition of NFkappaB in cancer cells converts inflammation- induced tumor growth mediated by TNFalpha to TRAIL-mediated tumor regression. Cancer Cell. 2004;6(3):297-305.

9. Troost E, Hold GL, Smith MG, Chow WH, Rabkin CS, McColl $\mathrm{KE}$, et al. The role of interleukin-1beta and other potential genetic markers as indicators of gastric cancer risk. Can $\mathrm{J}$ Gastroenterol. 2003 Jun;17 Suppl B:8B-12B.

10. Mocellin S, Rossi CR, Pilati P, Nitti D. Tumor necrosis factor, cancer and anticancer therapy. Cytokine Growth Factor Rev. 2005; 16(1):35-53.

11. Cozar JM, Canton J, Tallada M, Concha A, Cabrera T, Garrido F, et al. Analysis of NK cells and chemokine receptors in tumor infiltrating CD4 $\mathrm{T}$ lymphocytes in human renal carcinomas. Cancer Immunol Immunother. 2005 Sep;54(9):858-866.

12. Landis SH, Murray T, Bolden S, Wingo PA. Cancer statistics, 1998. CA Cancer J Clin. 1998 Jan-Feb;48(1):6-29.

13. Peggs KS, Quezada SA, Korman AJ, Allison JP. Principles and use of anti-CTLA4 antibody in human cancer immunotherapy. Curr Opin Immunol. 2006 Apr;18(2):206-13.

14. Lech-Maranda E, Baseggio L, Bienvenu J, Charlot C, Berger F, Rigal D, et al. Interleukin-10 gene promoter polymorphisms influence the clinical outcome of diffuse large B-cell lymphoma. Blood. 2004 May 1;103(9):3529-3534..

15. Mocellin S, Marincola FM, Young HA. Interleukin-10 and the immune response against cancer: A counterpoint. J Leukoc Biol 2005;78(5):1043-1051. 
16. Hussain SP, Hofseth LJ, Harris CC. Radical causes of cancer. Nat Rev Cancer 2003;3(4):276-285.

17. Wilson AG, Symons JA, McDowell TL, McDevitt HO, Duff GW. Effects of a polymorphism in the human tumor necrosis factor a promoter on transcriptional activation. Proc Natl Acad Sci U S A 1997,94(7):3195-3199.

18. Mocellin S, Rossi CR, Pilati P, Nitti D. Tumor necrosis factor, cancer and anticancer therapy. Cytokine Growth Factor Rev 2005, 16(1):35-53.

19. Mulé JJ, Custer M, Averbook B, Yang JC, Weber JS, Goeddel DV et al. RANTES secretion by gene-modified tumor cells results in loss of tumorigenicity in vivo: role of immune cell subpopulations. Hum Gene Ther 1996,7(13):1545-1553.

20. Duell EJ, Casella DP, Burk RD, Kelsey KT, Holly EA. Inflammation, genetic polymorphisms in proinflammatory genes TNF- $\alpha$, RANTES, and CCR5, and risk of pancreatic adenocarcinoma. Cancer Epidemiol Biomarkers Prev. 2006 Apr; 15 (4):726-731.

21. Liu H, Chao D, Nakayama EE, Taguchi H, Goto M, Xin X et al. Polymorphism in RANTES chemokine promoter affects HIV-1 disease progression. Proc Natl Acad Sci U S A 1999,96(8):45814585.

22. Dinarello CA. The interleukin-1 family: 10 years of discovery. FASEB J 1994,8(15):1314-1325.

23. El-Omar EM, Carrington M, Chow WH, McColl KE, Bream JH, Young HA et al. Interleukin-1 polymorphisms associated with increased risk of gastric cancer. Nature 2000,404(6776):398402.

24. Wang Y, Kato N, Hoshida Y, Yoshida H, Taniguchi H, Goto T et al. Interleukin-1beta gene polymorphisms associated with $\mathrm{h}$ epatocellular carcinoma in hepatitis $\mathrm{C}$ virus infection. Hepatology 2003,37:65-71.

25. Zienolddiny S, Ryberg D, Maggini V, Skaug V, Canzian F, Haugen A. Polymorphisms of the interleukin-1 beta gene are associated with increased risk of non-small cell lung cancer. Int J Cancer 2004,109(3):353-356.

26. Grimm C, Berger I, Tomovski C, Zeillinger R, Concin N, Leodolter $\mathrm{S}$ et al. A polymorphism of the interleukin-1 receptor antagonist plays a prominent role within the interleukin-1 gene cluster in vulvar carcinogenesis. Gynecol Oncol 2004,92(3): 936-940.
27. Sehouli J, Mustea A, Koensgen D, Chen FC, Lichtenegger W. Interleukin-1 receptor antagonist gene polymorphism is associated with increased risk of epithelial ovarian cancer Ann Oncol 2003,14(10):1501-1504.

28. Yoshimura T, Robinson EA, Tanaka S, Appella E, Kuratsu J, Leonard EJ. Purification and amino acid analysis of two human glioma-derived monocyte chemoattractants. J Exp Med 1989, 169(4): 1449-1459.

29. Roth SJ, Carr MW, Springer TA. C-C chemokines, but not the C-X-C chemokines interleukin-8 and interferon-gamma inducible protein-10, stimulate transendothelial chemotaxis of $\mathrm{T}$ lymphocytes. Eur J Immunol 1995,25(12):3482-488.

30. Ghilardi G, Biondi ML, La Torre A, Battaglioli L, Scorza R. Breast cancer progression and host polymorphisms in the chemokine system: role of the macrophage chemoattractant protein-1 (MCP-1) -2518 G allele. Clin Chem 2005,51(2):452-455.

31. Saji H, Koike M, Yamori T, Saji S, Seiki M, Matsushima K et al. Significant correlation of monocyte chemoattractant protein-1 expression with neovascularization and progression of breast carcinoma. Cancer 2001,92(5):1085-1091.

32. Rueda B, Zhernakova A, Lopez-Nevot MA, Gomez-Garcia M, Ortega E, Pinero A, et al. CTLA4/CT60 polymorphism is not relevant in susceptibility to autoimmune inflammatory intestinal disorders. Hum Immunol 2005;66(3):321-325.

33. Ueda H, Howson JM, Esposito L, Heward J, Snook H, Chamberlain G, et al. Association of the T-cell regulatory gene CTLA4 with susceptibility to autoimmune disease. Nature. 2003 May 29;423(6939):506-511.

34. Maurer M, Loserth S, Kolb-Maurer A, Ponath A, Wiese S, Kruse $\mathrm{N}$, et al. A polymorphism in the human cytotoxic T-lymphocyte antigen 4 ( CTLA4) gene (exon $1+49$ ) alters T-cell activation. Immunogenetics 2002;54(1):1-8.

Correspondencia autor: Dr. José M. Cózar Olmo Servicio de Urología. Hospital Univ. Virgen de las Nieves Avenida de las Fuerzas Armadas s/n - 18014 Granada E-mail autor: cozarjm@yahoo.es Información artículo: Original

Trabajo recibido: marzo 2009

Trabajo aceptado: abril 2009 